\title{
Adaptation of two types of processing gains for UWB impulse radio wireless sensor networks
}

\author{
i. Güvenç, H. Arslan, S. Gezici and H. Kobayashi
}

\begin{abstract}
Ultrawideband impulse radio systems offer two kinds of processing gains that can be adapted based on the interference level in the system so that quality of service requirements are fulfilled. An adaptive assignment scheme for two types of multiple-access parameters in clusterbased wireless sensor networks is investigated. A mathematical framework is developed for asynchronous communications using a Gaussian approximation method to model the multiple-access interference in two cases: one with fixed frame duration, where the goal is to increase the average throughput, and the other with fixed symbol duration, where the goal is to increase the network lifetime. Extension of the analysis to multipath channels is carried out, and the validity of the Gaussian approximation is investigated using the Kullback-Leibler distance.
\end{abstract}

\section{Introduction}

Ultrawideband impulse radio (UWB-IR) is a highly promising physical layer technology for wireless sensor networks (WSNs) due to its unique characteristics such as low-power transmission, low cost and low-complexity transceiver circuitry, flexibility to transmit within a large unlicensed spectrum (under certain regulations), precise location capability and secure transmission due to employed multiple-access sequences. In a UWB-IR system, time-hopping (TH) codes are employed as a multiple-access method [1]. By appropriately designing the $\mathrm{TH}$ codes, it is possible to control the multiple-access interference (MAI) in UWB systems to a certain extent $[2,3]$. The $\mathrm{TH}$ multiple-access can provide interference-free communications in synchronous systems. Even in an asynchronous system, excessive interference can be avoided due to low duty cycle and large processing gain (PG) of UWB-IR pulse transmission.

Adaptation of wireless communication systems allows better exploitation of the system resources based on the estimation of wireless link quality [4]. The link quality is often measured by the signal-to-interference-plus-noise ratio (SINR) of the received signal. For example, adaptive coding $[5,6]$ schemes can achieve higher throughput when the channel quality is good by decreasing the amount of redundancy transmitted (or increasing the modulation order). In contrast, when the link quality is poor, reliable transmission can be insured by increasing the amount of redundancy and coding

(C) The Institution of Engineering and Technology 2007

doi:10.1049/iet-com:20045255

Paper first received 15th October 2004 and in revised form 29th December 2006

I. Güvenç is with the DoCoMo USA Labs, 3240 Hillview Avenue, Palo Alto, CA 94304, USA

H. Arslan is with the Electrical Engineering Deptartment, University of South Florida, 4202 E. Fowler Ave., ENB-118, Tampa, FL 33620, USA

S. Gezici is with the Department of Electrical and Electronics Engineering, Bilkent University, Bilkent, Ankara 06800, Turkey

H. Kobayashi is with the Department of Electrical Engineering, Princeton University, Princeton, NJ 08544, USA

E-mail: iguvenc@eng.usf.edu power (or by decreasing the modulation order). Assigning multiple codes to the users, changing the pulse shape [7] and duration and the transmitted pulse power [8] as in the conventional schemes are other forms of adaptation in UWB systems to better exploit the system resources.

Adaptation of multiple-access parameters in UWB-IR systems is another flexible means of exploiting system resources efficiently. Unlike many other technologies such as direct-sequence code division multiple-access (DS-CDMA) systems, UWB-IR offers two different types of PGs: the number of pulses per symbol and the frame duration. Increasing the number of pulses per symbol increases the SINR, which can be considered as a power control approach in the time domain without changing the pulse amplitudes. Increasing the frame duration (which is related to the cardinality of the code) again improves the SINR in a multiuser environment, as it becomes less likely that the pulses will be corrupted. By measuring the link quality (which depends on the channel, multiuser interference etc.), it is possible to improve the data rate by modifying these two different multi-access parameters, while satisfying a minimum bit error rate (BER) requirement set by quality of service (QoS) requirements. Alternatively, if a certain data rate is required by the system, by adjusting these two PGs, the transmission power can be reduced to improve the network lifetime, when the link quality is good.

Adaptive rate and power allocation have been well studied for CDMA systems in the past [9-12]. Optimal assignment of number of pulses per symbol and the frame duration for UWB systems in range limited and multiuser interference-limited environments were analysed in [13], where the Gaussian approximation (GA) is used to characterise the link quality and assess data rate gains for asynchronous communications. In [14], the use of the standard Gaussian approximation to capture the MAI in power unbalanced scenarios was investigated, and it was shown to be applicable to densely deployed networks. Another GA of MAI for chip-synchronous and chipasynchronous scenarios was derived in [15] for a system with fixed number of pulses per symbol and fixed frame duration. Although adaptation of frame duration and number of pulses per symbol was analysed in [16] in the context of medium-access control for UWB ad hoc networks, a 
mathematical framework for the MAI has not been developed. In $[17,18]$, the radio-resource allocation problem was analysed as a theoretical constrained optimisation problem for ad hoc networks, where the system throughput is maximised considering a UWB physical layer, traffic patterns and system topology. Both reserved bandwidth (RB) (QoS) and dynamic bandwidth (best effort) scenarios are considered, and admission policies of new users to the system are presented.

In this paper, adaptation of multiple-access parameters is investigated in asynchronous environments for cluster based WSNs. Multiuser interference is modelled by a GA approach for two communication scenarios: fixed frame duration, where the goal is to maximize the overall data rate, and fixed symbol duration, where the goal is to have an identical data rate for all the users, and improve the network lifetime. For the fixed symbol duration case, the required symbol energy to meet the BER requirement is calculated, and the number of pulses to transmit is evaluated; this implies joint assignment of both the number of pulses per symbol and the frame duration, as the symbol duration is constant. Extension of the analysis to multipath channels is performed for both cases. Also, the validity of the GA for different parameters is evaluated using the Kullback-Leibler (KL) distance metric, and its effects on the BER is analysed for different parameters and SINRs. Improvements in the data rate and power consumption for the adaptation schemes are demonstrated with computer simulations for fixed and mobile cluster-head $(\mathrm{CH})$ scenarios. The main contributions of the paper can be summarised as follows: (1) derivation of an asymptotic closed-form expression for the probability distribution of MAI in a UWB-IR system with various frame durations for different users, (2) evaluation of the asymptotic study by a metric-based approach for various PG parameters and (3) a PG adaptation framework for UWB-IR WSNs based on the asymptotic analysis.

The paper is organised as follows. Section 2 gives the system model for the UWB signalling and the sensor network. Adaptation schemes for asynchronous communications systems are analysed in Section 3 and extensions to multipath channels are provided in Section 4. The validity of GA is investigated in Section 5 which is followed by the simulation results in Section 6. Finally, some concluding remarks are made.

\section{System model}

\subsection{UWB signal model}

In this section, a generic UWB signal model is introduced, where a variable number of pulses per symbol and variable frame durations are allowed for different users. The transmitted UWB signal from user $k$ in an $N_{\mathrm{u}}$ user system is given by

$$
s_{k}(t)=\sqrt{E_{\mathrm{tp}}^{(k)}} \sum_{j=-\infty}^{\infty} a_{j}^{(k)} b_{\left\lfloor j / N_{\mathrm{s}}^{(k)}\right\rfloor}^{(k)} \omega_{t x}\left(t-j T_{\mathrm{f}}^{(k)}-c_{j}^{(k)} T_{\mathrm{c}}\right)
$$

where $T_{\mathrm{f}}^{(k)}$ is the frame duration of user $k, j$ is the frame index, $T_{\mathrm{c}}$ the chip duration, $\omega_{t x}(t)$ the transmitted UWB pulse with unit energy and $E_{\mathrm{tp}}^{(k)}$ is the transmitted pulse energy for user $k$. The number of frames/pulses per information bit for user $k$ is denoted as $N_{\mathrm{s}}^{(k)}=T_{\mathrm{s}}^{(k)} / T_{\mathrm{f}}^{(k)}$, where $T_{\mathrm{s}}^{(k)}$ is the symbol period for user $k$, and the number of chips per frame of user $k$ is denoted by $N_{\mathrm{h}}^{(k)}$. The random polarity codes $a_{j}^{(k)}$ are binary random variables taking values \pm 1 with equal probability, and $a_{j}^{(k)}$ and $a_{i}^{(l)}$ are independent for $(k, j) \neq(l, i)$ [19]. Also, $c_{j}^{(k)} \in\left\{0,1, \ldots, N_{\mathrm{h}}^{(k)}-1\right\}$ with equal probability and $c_{j}^{(k)}$ and $c_{i}^{(l)}$ are independent for $(k, j) \neq(l, i)$. The transmitted bits of user $k$ are denoted by $b_{\left\lfloor j / N_{\mathrm{s}}^{(k)}\right\rfloor}^{(k)} \in\{-1,+1\}$.

The received signal over an AWGN channel is given by

$$
\begin{aligned}
r(t)= & \sum_{k=1}^{N_{\mathrm{u}}} \sqrt{E_{\mathrm{rp}}^{(k)}} \sum_{j=-\infty}^{\infty} a_{j}^{(k)} b_{\left\lfloor j / N_{\mathrm{s}}^{(k)}\right\rfloor}^{(k)} \omega_{r x}\left(t-j T_{\mathrm{f}}^{(k)}\right. \\
& \left.-c_{j}^{(k)} T_{\mathrm{c}}-\tau_{k}\right)+\sigma_{n} n(t)
\end{aligned}
$$

where $E_{\mathrm{rp}}^{(k)}$ is the received pulse energy, $\tau_{k}$ the delay of user $k, \omega_{r x}$ the received UWB pulse and $n(t)$ a zero-mean white Gaussian noise process with unit spectral density.

Consider a matched filter (MF) receiver, as shown in Fig. 1, with the following template signal for the zeroth bit of user $\xi\left(b_{0}^{(\xi)}\right)$, without loss of generality

$$
s_{\text {temp }}^{(\xi)}(t)=\frac{1}{\sqrt{N_{\mathrm{s}}^{(\xi)}}} \sum_{j=0}^{N_{\mathrm{s}}^{(\xi)}-1} a_{j}^{(\xi)} \omega_{r x}\left(t-j T_{\mathrm{f}}^{(\xi)}-c_{j}^{(\xi)} T_{\mathrm{c}}-\tau_{\xi}\right)
$$

Then, the output of the MF is given by

$$
Y=b_{0}^{(\xi)} \sqrt{E_{\mathrm{rp}}^{(\xi)} N_{\mathrm{s}}^{(\xi)}}+M+N
$$

where $N \sim N\left(0, \sigma_{n}^{2}\right)$ is the output noise and $M$ the total MAI, which is the sum of interference terms from the interfering users

$$
M=\sum_{k=1, k \neq \xi}^{N_{\mathrm{u}}} M_{k}
$$

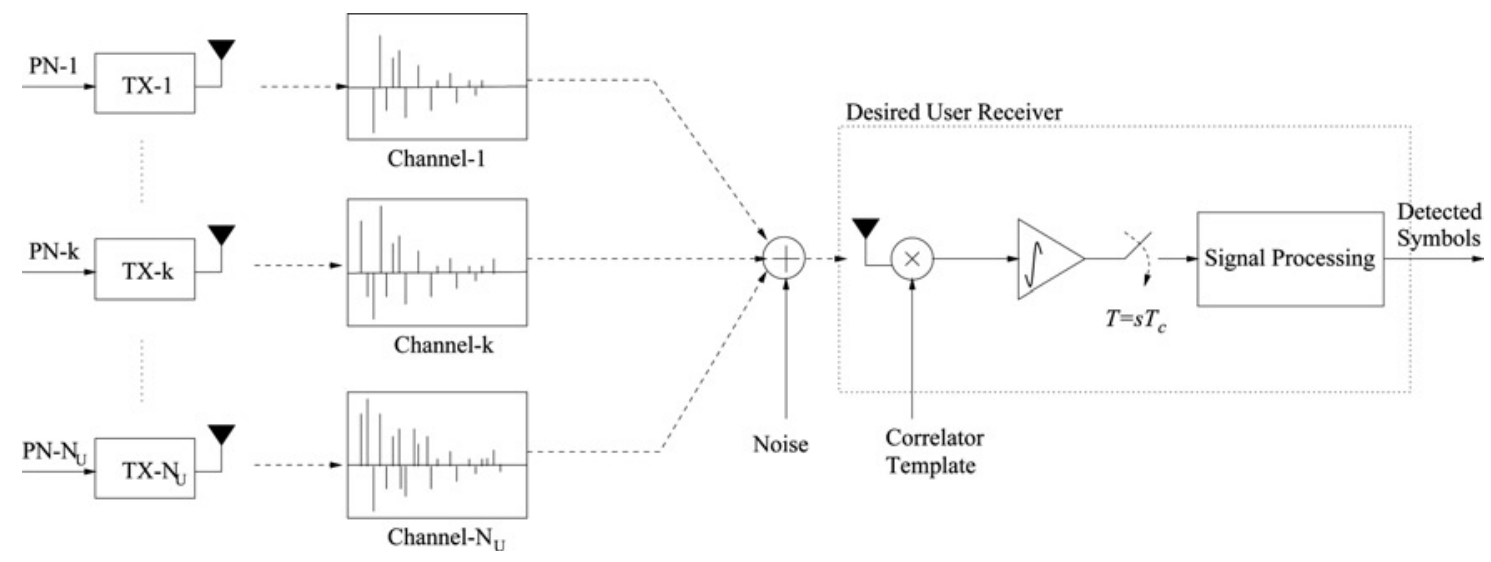

Fig. 1 Received signals from multiple users and the correlator receiver 
where $M_{k}$ is the MAI from user $k$. The statistics of $M$ will be analysed in Section 3.

\subsection{Sensor network model and BER evaluation}

A cluster-based WSN is considered, where the $\mathrm{CH}$ has more complex circuitry, and therefore higher processing capabilities compared with the sensor nodes. In general, the $\mathrm{CH}$ may also be selected from one of the sensor nodes as in [20]. However, this may increase the overall complexity of the network since larger complexity of the $\mathrm{CH}$ will be required for each individual sensor node. In contrast, the adaptation scheme to be introduced can be applied to other sensor network architectures with centralised control. The communication happens in rounds as in [20], where, after each round, the $\mathrm{CH}$ may update the multiple-access parameters. Consider a cluster of $N_{\mathrm{u}}$ sensors, with the $k$ th node having a transmitted pulse energy of $E_{\mathrm{tp}}^{(k)}$ to communicate with the $\mathrm{CH}$, which transmits the information to a remote base station. The received pulse energy for user $k$ at the $\mathrm{CH}$ is given by

$$
E_{\mathrm{rp}}^{(k)}=E_{\mathrm{tp}}^{(k)} \frac{\alpha_{k}}{d_{k}^{n}}
$$

where $n$ denotes the path loss exponent, $d_{k}$ the distance between the $k$ th sensor node and the $\mathrm{CH}$, and $\alpha_{k}$ the fading coefficient for user $k$. When there is no MAI, the probability of error for user $k$ which employs binary phase shift keying (BPSK) modulation is given by

$$
P_{\mathrm{b}}^{(k)}=Q\left(\sqrt{\mathrm{SNR}_{k}}\right)=Q\left(\sqrt{\frac{N_{\mathrm{s}}^{(k)} E_{\mathrm{rp}}^{(k)}}{\sigma_{n}^{2}}}\right)
$$

where energy per symbol (bit) of user $k$ is given by $E_{\mathrm{rs}}^{(k)}=N_{\mathrm{s}}^{(k)} E_{\mathrm{rp}}^{(k)}, Q(x)$ is given by $1 / 2(\operatorname{erfc})(x / \sqrt{2})$ and SNR denotes the signal-to-noise ratio (interference effects will be considered later). Conventional UWB networks use the same number of pulses per symbol and the same frame duration for each user, ensuring reliable communications with the user that has the worst-link quality. If the minimum BER required by the system is given by $P_{\mathrm{b}}$, the PG assigned to each user is given by

$$
N_{\mathrm{s}}=\frac{\left[Q^{-1}\left(P_{\mathrm{b}}\right)\right]^{2} \sigma_{n}^{2}}{E_{\mathrm{rp}}^{\min }}
$$

where $E_{\mathrm{rp}}^{\min }$ denotes the minimum received pulse energy, which is from the furthest away user in an ideal environment. The raw data rate for each user is then given by $1 /\left(N_{\mathrm{s}} N_{\mathrm{h}} T_{\mathrm{c}}\right)$.
In order to better exploit the system resources, it is possible to change the number of pulses $\left(N_{\mathrm{s}}^{(k)}\right)$, and number of chips per frame $\left(N_{\mathrm{h}}^{(k)}\right)$, for each user based on the channel quality, the distance of the user from the $\mathrm{CH}$, the longand short-term fading effects and the interference level in the system. In [3], a synchronous scenario is investigated where the orthogonal construction of $\mathrm{TH}$ sequences allows interference-free communications, such as in the downlink. However, the synchronous signalling is not very practical for WSNs, in general, and hence we focus on the asynchronous scenario. In the next section, the adaptation of $N_{\mathrm{s}}^{(k)}$ and $N_{\mathrm{h}}^{(k)}$ in asynchronous systems is analysed under a BER constraint for two different cases: fixed frame duration (to maximise the data rate) and fixed symbol duration (to maximise the network lifetime).

\section{Parameter adaptation for asynchronous communications}

In order to calculate the BER of the desired user in the presence of multiple users with random $\mathrm{TH}$ codes, we employ a GA approach for large number of pulses per information symbol. This is similar to the GAs employed in [15, 19]. However, we derive a more generic expression that is valid for variable numbers of frame sizes and covers the results in $[15,19]$ as a special case.

For analytical purposes, we approximate an asynchronous UWB system by a chip-synchronous system, where the misalignment between the symbols of the users is integer multiples of the chip interval $T_{\mathrm{c}}$. Assuming without loss of generality that the delay of the desired user is zero $\left(\tau_{\xi}=0\right), \tau_{k}=\Delta_{k} T_{\mathrm{c}}$ for $k \neq \xi$, where $\Delta_{k} \in\left\{0,1, \ldots, N_{\mathrm{h}}^{(k)} N_{\mathrm{s}}^{(k)}-1\right\}$ with equal probability. As studied in [15], the chip-synchronous assumption usually results in over-estimating the error probability in random TH UWB-IR systems, and hence the system design based on this approximation is usually on the safe side.

\subsection{Case 1: fixed throughput (variable frame duration)}

Consider the case where a fixed throughput is to be assigned to all users. Hence, we consider a common symbol time and BER in this scenario. In other words, the total PG, defined by $N_{\mathrm{c}}=N_{\mathrm{s}}^{(k)} N_{\mathrm{h}}^{(k)}$, is constant in this case [see Fig. $2 b$, where $\left(N_{\mathrm{s}}^{(1)}, N_{\mathrm{h}}^{(1)}\right)=(3,4),\left(N_{\mathrm{s}}^{(2)}, N_{\mathrm{h}}^{(2)}\right)=(4,3), \quad$ and $\left.\left(N_{\mathrm{s}}^{(3)}, N_{\mathrm{h}}^{(3)}\right)=(6,2)\right]$. Therefore the number of pulses per symbol and the frame duration can be changed as long as their multiplication is fixed. In this case, the following lemma is employed in order to approximate the MAI from user $k$.

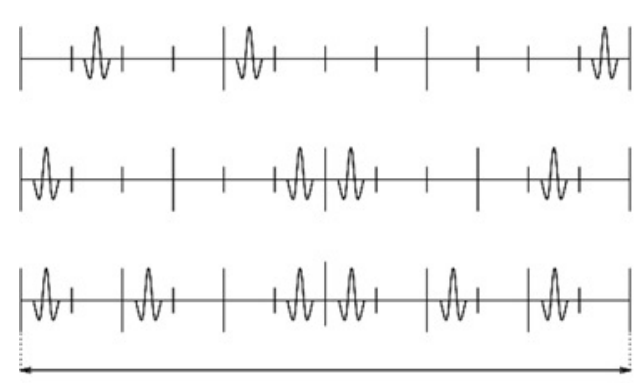

a

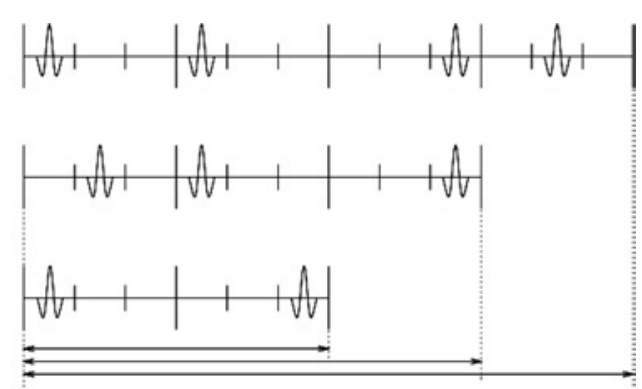

$b$

Fig. 2 Example transmitted signals

$a$ Symbol durations for fixed frame duration

$b$ Symbol durations for fixed throughput 
Lemma 1: In a chip-synchronous scenario, the distribution of the MAI from user $k$ converges to the following Gaussian random variable

$$
M_{k} \sim \mathcal{N}\left(0, \frac{E_{\mathrm{rp}}^{(k)}}{N_{\mathrm{h}}^{(k)}}\right)
$$

as $\min \left\{N_{\mathrm{s}}^{(\check{\xi})}, N_{\mathrm{s}}^{(k)}\right\} \rightarrow \infty$

\section{Proof: See Appendix A.}

In other words, for large values of $N_{\mathrm{s}}^{(\xi)}$ and $N_{\mathrm{s}}^{(k)}$, the MAI from user $k$ converges to a zero-mean Gaussian random variable. Note that the expression in (9) reduces to the result in [15] for $N_{\mathrm{h}}^{(k)}=N_{\mathrm{h}}^{(\xi)} \forall k$.

From (9), the total MAI can be approximated as

$$
M \sim \mathcal{N}\left(0, \sum_{k=1, k \neq \xi}^{N_{\mathrm{u}}} \frac{E_{\mathrm{rp}}^{(k)}}{N_{\mathrm{h}}^{(k)}}\right)
$$

Then, the SINR of the system can be obtained as

$$
\mathrm{SINR}=\frac{N_{\mathrm{s}}^{(\xi)} E_{\mathrm{rp}}^{(\xi)}}{\sigma_{n}^{2}+\sum_{\substack{k=1 \\ k \neq \xi}}^{N_{\mathrm{u}}} E_{\mathrm{rp}}^{(k)} / N_{\mathrm{h}}^{(k)}}
$$

which can be expressed as

$$
\mathrm{SINR}=\frac{E_{\mathrm{rs}}^{(\xi)}}{\sigma_{n}^{2}+\left(1 / N_{\mathrm{c}}\right) \sum_{\substack{k=1 \\ k \neq \xi}}^{N_{\mathrm{u}}} E_{\mathrm{rs}}^{(k)}}
$$

by defining the received symbol energy of the $k$ th user by $E_{\mathrm{rs}}^{(k)}=N_{\mathrm{s}}^{(k)} E_{\mathrm{rp}}^{(k)}$ for $k=1, \ldots, N_{\mathrm{u}}$.

When the same SINR value is assigned to all the users, they have the same BER, hence the same throughput, as they have the same symbol time. Hence, from (12), it is observed that the same received symbol energy can be used to achieve the same BER for all users. The common energy, denoted by $E_{\mathrm{rs}}$, can be obtained from (12) as

$$
E_{\mathrm{rs}}=\frac{\sigma_{n}^{2} \mathrm{SINR}}{1-\left(\left(N_{\mathrm{u}}-1\right) / N_{\mathrm{c}}\right) \mathrm{SINR}}
$$

In other words, for a desired SINR value, the required received symbol energy of the users can be calculated. Since the symbol energy is the multiplication of the number of pulses per symbol and the pulse energy, the received symbol energy can be expressed as

$$
E_{\mathrm{rs}}=E_{\mathrm{ts}}^{(k)} \frac{\alpha_{k}}{d_{k}^{n}}=N_{\mathrm{s}}^{(k)} E_{\mathrm{tp}}^{(k)} \frac{\alpha_{k}}{d_{k}^{n}}
$$

Therefore the users can use different numbers of pulses per symbol and/or different pulse energies depending on the channel state and their location. In a practical setting, the $\mathrm{CH}$ can calculate the SINR for each of the users and feedback them how to scale their symbol energy in order to achieve the desired SINR. Note that when a user is very far away from the $\mathrm{CH}$ or its channel is in a deep fade, the transmitted symbol energy needs to be increased considerably, which might violate the FCC's regulations [21]. Therefore multi-hopping might be necessary in some cases.

The received signal energy in (14) implies that given the fading coefficient and distance of user $k$, the energy can be set by changing $N_{\mathrm{s}}^{(k)}$ and/or $E_{\mathrm{tp}}^{(k)}$. In other words, there is a flexibility in adjusting the symbol energy. Note that this is different from the RB case in [17], since $N_{\mathrm{s}}^{(k)}$ and $N_{\mathrm{h}}^{(k)}$ are both variable (their multiplication is constant) in our case.
In [17], the RB case assumes that $N_{\mathrm{s}}^{(k)}$ is fixed (implying that $N_{\mathrm{h}}^{(k)}$ is fixed as the data rate is fixed), and therefore the adaptation is acquired by only scaling $E_{\mathrm{tp}}^{(k)}$.

Even though there is a flexibility in adjusting the received power, there are a few issues to consider when setting the symbol energy. First, the FCC's implicit limitation on the peak-to-average signal ratio can restrict the use of very small $N_{\mathrm{s}}^{(k)}$ values. Secondly, the inter-frame interference (IFI) can be an issue in a multipath environment when choosing the number of frames per symbol, where choosing the larger frames reduces the effects of the IFI.

\subsection{Case 2: fixed frame duration}

In this case, the frame durations of all the users are the same. Hence, $N_{\mathrm{h}}$ is common for all of them (see, e.g. Fig. $2 a$, where $N_{\mathrm{s}}^{(1)}=4, N_{\mathrm{s}}^{(2)}=3, N_{\mathrm{s}}^{(3)}=2$ and $N_{\mathrm{h}}^{(k)}=3$ for all $k$ ).

The aim is to meet the BER requirement for all users in the system. In order to satisfy a certain BER threshold, the number of pulses per symbol is adapted in order to maximise the overall data rate of the system [13].

The GA approach in the previous case can directly be applied to the fixed frame duration case in which $N_{\mathrm{h}}^{(k)}=N_{\mathrm{h}} \forall k$ (This special case is also investigated in [15].). Then, the MAI from user $k$ can be approximated by the following Gaussian random variable, when the number of pulses per information symbol for user $\xi, N_{\mathrm{s}}^{(\xi)}$, is large

$$
M_{k} \sim \mathcal{N}\left(0, \frac{E_{\mathrm{rp}}^{(k)}}{N_{\mathrm{h}}}\right)
$$

where $E_{\mathrm{rp}}^{(k)}$ is the energy of a received pulse from user $k$.

From (15), (4) and (5), the SINR of the system for user $\xi$ can be expressed as

$$
\mathrm{SINR} \simeq \frac{N_{\mathrm{s}}^{(\xi)} E_{\mathrm{rp}}^{(\xi)}}{\sigma_{n}^{2}+\left(1 / N_{\mathrm{h}}\right) \sum_{\substack{\mathrm{u}=1 \\ k \neq \xi}}^{N_{\mathrm{u}}} E_{\mathrm{rp}}^{(k)}}
$$

from which the value of $N_{\mathrm{s}}^{(\xi)}$ is obtained as

$$
N_{\mathrm{s}}^{(\xi)}=\left\lceil\frac{\operatorname{SINR}}{E_{\mathrm{rp}}^{(\xi)}}\left(\sigma_{n}^{2}+\frac{1}{N_{\mathrm{h}}} \sum_{\substack{k=1 \\ k \neq \xi}}^{N_{\mathrm{u}}} E_{\mathrm{rp}}^{(k)}\right)\right\rceil
$$

In other words, by setting the value of $N_{\mathrm{s}}^{(\xi)}$ according to (17), we transmit just enough number of pulses per symbol to meet the BER requirement. This is contrary to the conventional systems, where the worst-case parameters are used for all users, and hence a lower overall data rate is obtained. Note that all the users transmit with the same power over a block; however, for a given transmit power, the bit rate will depend on the link quality.

\section{Extensions to multipath channels}

Owing to the extremely short-duration pulses employed, it is likely to observe individual multipath components (although not very dispersive) even in low-power and very short-range communications in densely deployed sensor networks. Longer-range communications may yield much severe and dispersive channel impulse responses, where the maximum excess delay of the channel may be on the order of hundreds of nanoseconds. Therefore it becomes very crucial to consider the effects of multipath since it can have significant effects on the performance. 
Consider the transmission over frequency-selective channels, where the channel for user $k$ is modelled as

$$
h^{(k)}(t)=\sum_{l=1}^{L} \alpha_{l}^{(k)} \delta\left(t-(l-1) T_{\mathrm{c}}-\tau_{k}\right)
$$

where $\alpha_{l}^{(k)}$ and $\tau_{k}$ are the fading coefficient of the $l$ th path and the delay of user $k$, respectively, and $L$ the total number of received taps. Assume that $\tau_{1}=0$ and $\sum_{l=1}^{L}\left|\alpha_{l}^{(k)}\right|^{2}=1$, without loss of generality.

From (1) and (18), the received signal can be expressed as

$$
\begin{aligned}
r(t)= & \sum_{k=1}^{N_{\mathrm{u}}} \sqrt{E_{\mathrm{rp}}^{(k)}} \sum_{j=-\infty}^{\infty} a_{j}^{(k)} b_{\left\lfloor j / N_{\mathrm{f}}\right\rfloor}^{(k)} u^{(k)}\left(t-j T_{\mathrm{f}}^{(k)}\right. \\
& \left.-c_{j}^{(k)} T_{\mathrm{c}}-\tau_{k}\right)+\sigma_{n} n(t)
\end{aligned}
$$

where

$$
u^{(k)}(t)=\sum_{l=1}^{L} \alpha_{l}^{(k)} \omega_{r x}\left(t-(l-1) T_{\mathrm{c}}\right)
$$

Consider a Rake receiver for the $\xi$ th user, which has the following template signal for the 0 th information bit

$$
s_{\mathrm{temp}}^{(\xi)}(t)=\frac{1}{\sqrt{N_{\mathrm{s}}^{(\xi)}}} \sum_{j=0}^{N_{\mathrm{s}}^{(\xi)}-1} a_{j}^{(\xi)} v\left(t-j T_{\mathrm{f}}^{(\xi)}-c_{j}^{(\xi)} T_{\mathrm{c}}\right)
$$

where

$$
v(t)=\sum_{l=1}^{L} \beta_{l} \omega_{r x}\left(t-(l-1) T_{\mathrm{c}}\right)
$$

with $\boldsymbol{\beta}=\left[\beta_{1}, \ldots, \beta_{L}\right]$ being the Rake combining weights.

As considered in [22], the template signal given by (21) and (22) can represent different multipath diversity combining schemes by appropriate choices of the weighting vector $\boldsymbol{\beta}$.

From (19)-(22), the output of the Rake receiver can be expressed as follows

$$
\begin{aligned}
Y & =\int r(t) s_{\mathrm{temp}}^{(\xi)}(t) \mathrm{d} t \\
& =b_{0}^{(\xi)} \sqrt{N_{\mathrm{s}}^{(\xi)} E_{\mathrm{rp}}^{(\xi)}} \sum_{l=1}^{L} \alpha_{l}^{(\xi)} \beta_{l}+M+N
\end{aligned}
$$

where the first term is the desired signal part, $M$ the MAI from other users and $N$ is the output noise, which is approximately distributed as $N \sim \mathcal{N}\left(0, \sigma_{n}^{2} \sum_{l=1}^{L} \beta_{l}^{2}\right)$ for large $N_{\mathrm{s}}^{(\xi)}$ [22]. Assume $N_{\mathrm{h}}^{(\xi)} \gg(L-1)$ so that the IFI and the intersymbol interference are negligible [23].

The MAI term in (23) can be expressed as in (5); that is, as the sum of MAI terms from other users.

For the fixed throughput case, the following result can be obtained.
Lemma 2: In a chip-synchronous scenario, the distribution of the MAI from user $k$ converges to the following Gaussian random variable

$$
\begin{aligned}
M_{k} \sim N\left(0, \frac{E_{\mathrm{rp}}^{(k)}}{N_{\mathrm{h}}^{(k)}}\left[\sum_{j=1}^{L}\left(\sum_{l=1}^{j} \beta_{l} \alpha_{l+L-j}^{(k)}\right)^{2}\right.\right. \\
\left.\left.+\sum_{j=1}^{L-1}\left(\sum_{l=1}^{j} \alpha_{l}^{(k)} \beta_{l+L-j}\right)^{2}\right]\right)
\end{aligned}
$$

as $\min \left\{N_{\mathrm{s}}^{(\xi)}, N_{\mathrm{s}}^{(k)}\right\} \rightarrow \infty$.

Proof: See Appendix B.

Note that the result reduces to that in [22] for $N_{\mathrm{h}}^{(k)}=N_{\mathrm{h}}^{(\xi)}=N_{\mathrm{h}} \forall k$. This special case can be used for the fixed frame duration case to obtain the asymptotic MAI distribution as

$$
\begin{aligned}
M_{k} \sim \mathcal{N}\left(0, \frac{E_{\mathrm{rp}}^{(k)}}{N_{\mathrm{h}}}\left[\sum_{j=1}^{L}\left(\sum_{l=1}^{j} \beta_{l} \alpha_{l+L-j}^{(k)}\right)^{2}\right.\right. \\
\left.\left.+\sum_{j=1}^{L-1}\left(\sum_{l=1}^{j} \alpha_{l}^{(k)} \beta_{l+L-j}\right)^{2}\right]\right)
\end{aligned}
$$

for large $N_{\mathrm{s}}^{(\xi)}$.

From (24) and (25), it is observed that the MAI from an interfering user converges, as $N_{\mathrm{s}}^{(\xi)}$ and $N_{\mathrm{s}}^{(k)}$ become infinity, to Gaussian random variables with zero mean, similar to the ones in (9) and (15), respectively, with the only difference being the scaling factors to the variance terms, which purely depend on the multipath channel of the interfering user and the finger assignment of the Rake receiver. In other words, the same dependence on the received pulse energy and the PG parameters $\left(N_{\mathrm{s}}\right.$ and $\left.N_{\mathrm{h}}\right)$ is preserved as in the AWGN case.

\section{Validity of Gaussian approximation}

In the previous sections, GAs were used to model the multiuser interference in an asynchronous environment. In this section, the dependence of the accuracy of GA on the two types of PGs is analysed using the KL distance [24]. Moreover, the accuracy of the GA is evaluated for different multiple-access parameters and SNR values.

\subsection{KL distance between the approximate and actual MAl distributions}

Consider (9) and (15) for cases 1 and 2, respectively, where the interference from a second user was approximated using a Gaussian distribution with its variance depending on the parameters $N_{\mathrm{s}}^{(\xi)}, N_{\mathrm{h}}^{(k)}$ and $E_{\mathrm{rp}}^{(k)}\left(N_{\mathrm{h}}^{(k)}\right.$ is constant for case 2). In order to see how well the approximation captures the actual interference probability density function (PDF), the theoretical Gaussian PDF and the MAI PDF obtained from simulations can be compared for different ranges of multiple-access parameters. The KL distance (or relative entropy) is commonly used to characterise the similarity between two distributions. Let $f_{\text {theo }}^{N_{\mathrm{s}}^{(\xi)}, N_{\mathrm{h}}^{(k)}}$ denote the PDF of the interference corresponding to a set of parameters $N_{\mathrm{s}}^{(\xi)}$ and $N_{\mathrm{h}}^{(k)}$, and let $f_{\mathrm{sim}}^{N_{\mathrm{s}}^{(\xi)}, N_{\mathrm{h}}^{(k)}}$ denote the PDF of the interference 
generated using simulations and corresponding to the same set of parameters. Then, the KL distance between two distributions is given by

$$
K\left(f_{\text {theo }}^{N_{\mathrm{s}}^{(s)}, N_{\mathrm{h}}^{(k)}} \| f_{\mathrm{sim}}^{N_{\mathrm{s}}^{(\xi)}, N_{\mathrm{h}}^{(k)}}\right)=\sum_{i=-\infty}^{\infty} f_{\text {theo }}^{N_{\mathrm{s}}^{(\xi)}, N_{\mathrm{h}}^{(k)}}(i) \times \ln \frac{f_{\text {theo }}^{N_{\mathrm{s}}^{(\xi)}, N_{\mathrm{h}}^{(k)}}(i)}{f_{\mathrm{sim}}^{N_{\mathrm{s}}^{(\xi)}, N_{\mathrm{h}}^{(k)}}(i)}
$$

The larger the KL distance, the less would be the similarity between the two PDFs. As the KL distance metric is not symmetric, the average of the two KL distances (i.e. $K\left(f_{\text {theo }} \| f_{\text {sim }}\right)$ and $\left.K\left(f_{\text {sim }}|| f_{\text {theo }}\right)\right)$ is used in this paper to evaluate the similarity between the two distributions.

Note that while the interference distribution lies between $\left(-\sqrt{ }\left(E_{\mathrm{rp}}^{(\xi)} N_{\mathrm{s}}^{(\xi)}\right), \sqrt{ }\left(E_{\mathrm{rp}}^{(\xi)} N_{\mathrm{s}}^{(\xi)}\right)\right)$, the support of the theoretical Gaussian distribution is $(-\infty, \infty)$. Analysing (26) under this fact implies that KL distance may converge to infinity if not properly treated. Therefore as an approximation, we truncate the theoretical Gaussian distribution to lie within the support of the interference distribution, and the area under the omitted tails of the Gaussian distribution is included as delta functions at the edges of the truncated Gaussian distribution.

In Fig. 3, the simulation results for case 2 are presented for various values of frame durations and PGs. Two users with equal power levels are considered, and the KL distances are computed for different values of $N_{\mathrm{s}}^{(\xi)}$ and $N_{\mathrm{h}}^{(k)}$. It is observed that the MAI converges to a Gaussian distribution for larger values of $N_{\mathrm{s}}^{(\xi)}$ and for smaller values of $N_{\mathrm{h}}^{(k)}\left(2 \times 10^{7}\right.$ bits are used in simulations $)$. Similar simulations are repeated for case 1 , where similar results are observed.

\subsection{BER performances using the $G A$ and the actual MAl distribution}

Even though the KL distance characterises the accuracy of GA for different set of parameters (relative to another set of parameters), how much this will affect the BER is also dependent on SNR. For example, if the noise variance is large, inaccuracy of the GA may not yield significant deviations from the actual BER. In contrast the BER of the systems operating at high SNR

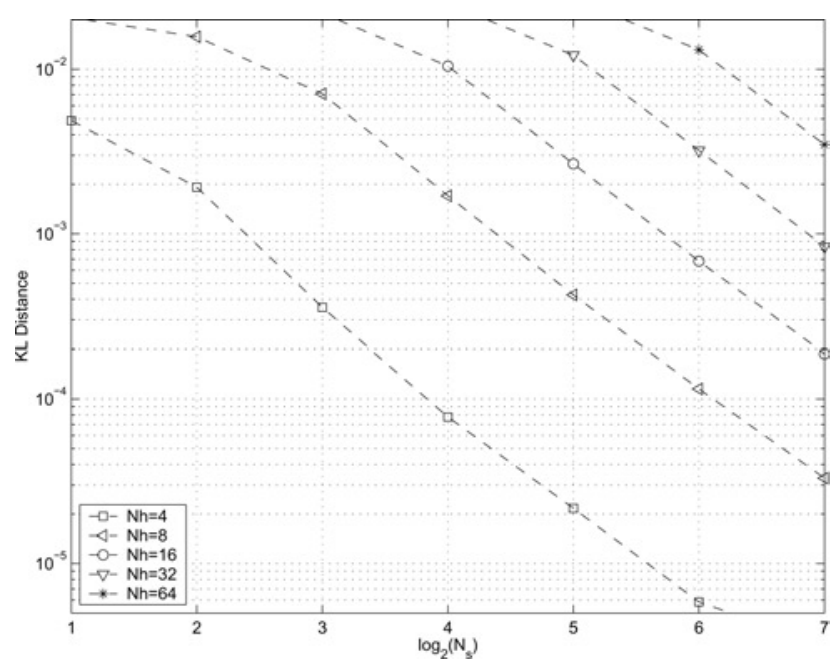

Fig. $3 K L$ distances for case 2 with respect to $N_{\mathrm{s}}^{(\xi)}$ and $N_{\mathrm{h}}^{(k)}$. Only two users with equal power are considered

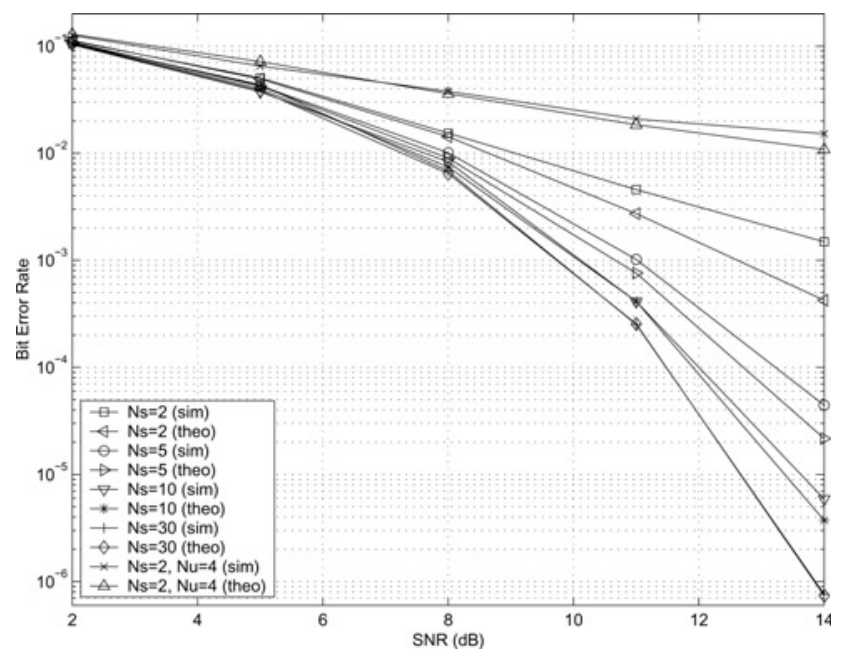

Fig. 4 Comparison of theoretical and simulation BERs for case 1. Only two users with equal power are considered $\left(N_{h}=10\right)$

environments may be very sensitive against inaccuracies in the GA.

In Fig. 4, the BER against SNR curves for case 2 and for different multiple-access parameters are presented, which are obtained using the simulations and the GA. It is observed that the larger values of $N_{\mathrm{s}}$ increases the accuracy of GA. It is also seen that as the SNR increases, the deviation between the BERs obtained using the GA and the simulations increases. The theoretical and simulation BER results for four users were also presented for comparison purposes, where it can be observed that GA provides a tighter bound.

\section{Simulation results}

Computer simulations are performed to demonstrate the improvements in the data rate and reductions in power consumption. Only a single cluster of a WSN is considered in the simulations, and 100 sensor nodes are randomly distributed over a $25 \times 25 \mathrm{~m}$ field. The results can also be generalised for multiple clusters, where sensor nodes in each cluster communicate adaptively with the $\mathrm{CH}$, and the $\mathrm{CH}$ (which form another upper-level cluster within themselves) communicate adaptively with the sink. Corresponding to a BER of $10^{-4}$ for BPSK modulation, $\mathrm{SNR}=8.39 \mathrm{~dB}$ is targeted. The path loss exponent is taken to be $n=2.4$, the pulse width is set to $T_{\mathrm{c}}=0.3 \mathrm{~ns}$, and the chip-synchronous case is considered in all scenarios. It is assumed that the transmitted pulse occupies the whole $7.5 \mathrm{GHz}$ of bandwidth in between 3.1 and $10.6 \mathrm{GHz}$. Since the FCC mask allows a maximum transmission power of $-41 \mathrm{dBm} / \mathrm{MHz}$ within this frequency range, the maximum transmit energy per second can be calculated to be $0.562 \mathrm{~mW}$. This is the maximum power that any sensor can transmit within the limits of FCC regulations, which might restrict the selection of optimum $N_{\mathrm{h}}$ and $N_{\mathrm{s}}$ even if SINR is appropriate.

Simulation results for asynchronous scenario of case 1 are presented in Figs. 5 and 6, where the data rates are identical for all the users: $\left(N_{\mathrm{c}} T_{\mathrm{c}}\right)^{-1}=\left(10^{4} \times 0.3 \times\right.$ $\left.10^{-9}\right)^{-1}=33 \mathrm{kbps}$, with $N_{\mathrm{c}}=N_{\mathrm{s}} \times N_{\mathrm{h}}=10^{4}$. For simulation purposes, continuous transmission of all the sensors and very low-initial battery energy assignments $(1 \mathrm{~mJ})$ for each node are assumed. The parameters are updated after 


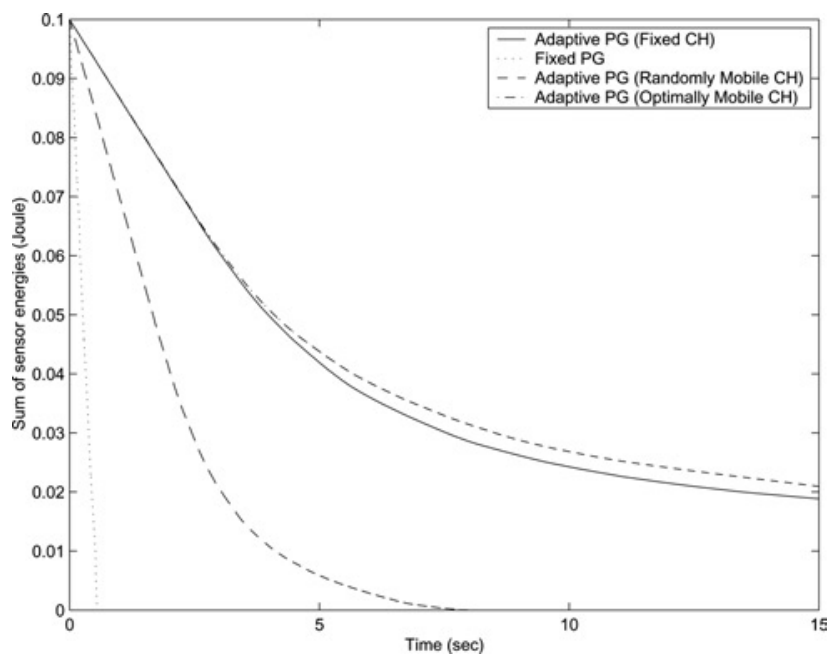

Fig. 5 Remaining aggregate energy in the network with respect to time

each round of $300 \mu \mathrm{s}$ to adapt to the Rayleigh-fading channel and possibly changed distances, and the energy consumption in $5 \times 10^{4}$ rounds is analysed. Simulation results indicate substantial gains in network lifetime when using adaptive assignment of PG. Also, the effects of mobility of the $\mathrm{CH}$ is analysed. This may be considered, for example, for rescue-robot applications where the robot acts as a $\mathrm{CH}$ to communicate with various sensors, and although the power consumption of the robot is not that crucial, we would like to maximise the network lifetime of the sensors. It is observed in Figs. 5 and 6 that if the $\mathrm{CH}$ randomly moves in the network, the network lifetime shortens seriously. On the other hand, the movement of the $\mathrm{CH}$ after each round to an optimal location (i.e. the expected value of the locations of the alive sensor nodes) slightly increases the network lifetime compared with the case when the $\mathrm{CH}$ is motionless and located at the centre of the network. In Fig. 7, we also compare (averaged over $10^{4}$ sensor realisations) the metric $T_{0.95}=T_{0.95}^{\text {(adapt) }} / T_{0.95}^{\text {(fixed) }}$ for different parameters, where $T_{0.95}^{\text {(adapt) }}$ and $T_{0.95}^{\text {(fixed) }}$ are the time durations where the total network energy falls to $95 \%$ of the initial network energy for adaptive PG and fixed PG cases, respectively. We observe that as the number of sensor nodes increases or the network

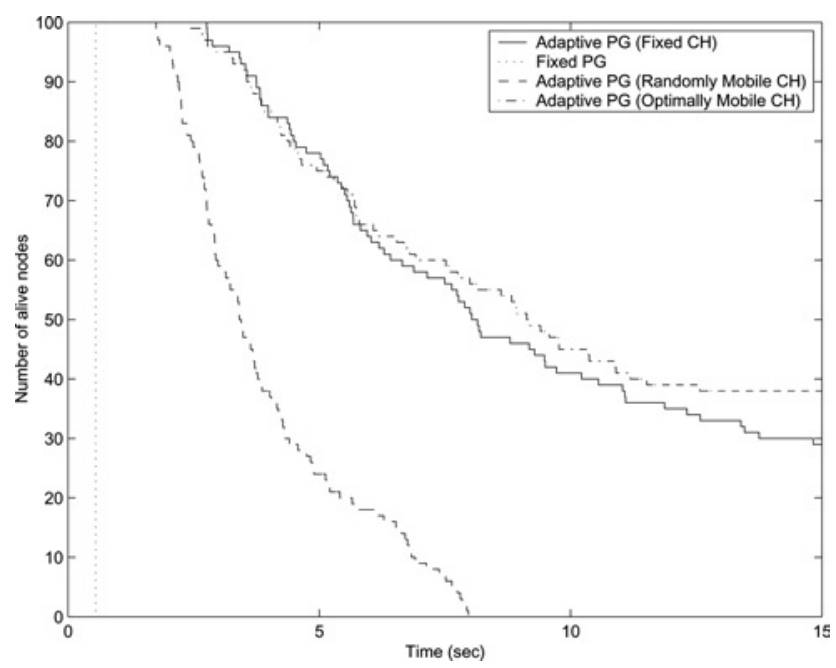

Fig. 6 Number of alive nodes in the network with respect to time

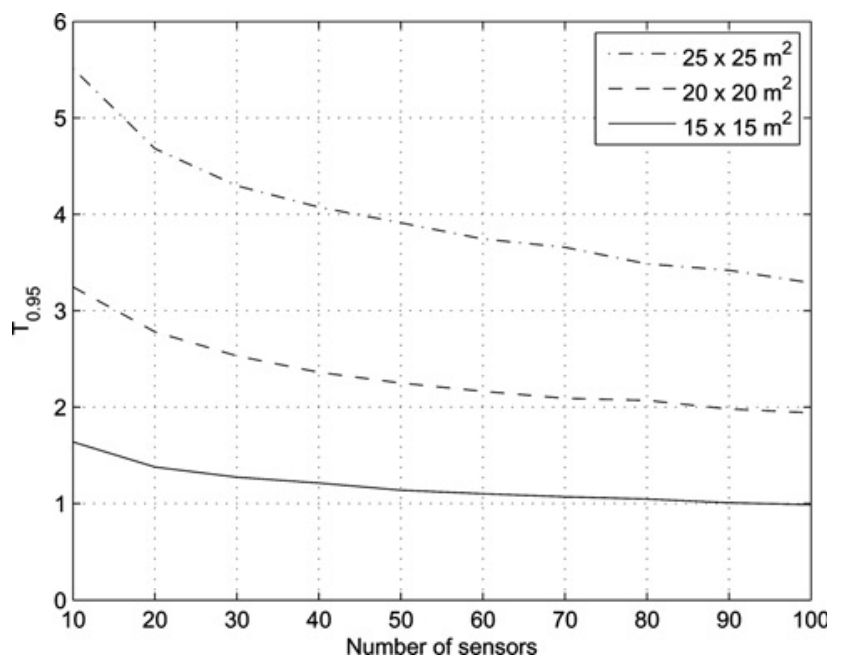

Fig. 7 Ratio of the time periods for the proposed and conventional techniques where the total network energy falls to 95\% of the initial network energy

dimensions decreases (i.e. the sensor intensity increases), the gains obtained from the adaptive $P G$ approach diminishes. In particular, for the $15 \times 15 \mathrm{~m}^{2}$ scenario, there is almost no gain. This implies that the proposed technique is less appropriate for short-range communications where the path loss is less severe. Nevertheless, as implied by Fig. 5, the adaptive PG approach will still have merit for longer observation windows.

\section{Conclusion}

In this paper, the adaptation of multiple-access parameters in cluster-based UWB-IR WSNs has been analysed. A GA method has been employed to adapt the transmission powers and the PGs of the sensors, and a mathematical framework has been developed for the analysis of MAI when the users employ different numbers of pulses per symbol and different frame durations. The main contribution of the paper is on the analysis of variable frame duration case, both in AWGN and in multipath channels. Also, the accuracy of the GA has been investigated and quantified using the KL distance based on the parameters $\left(N_{\mathrm{s}}, N_{\mathrm{h}}\right)$ in a way not addressed in the literature before. It has been shown to be accurate for populated networks with large $N_{\mathrm{s}}$, small $N_{\mathrm{h}}$, and low SNR values. Simulation results outline the potential improvements in energy savings using the adaptive system design based on the two parameters.

Many of the analysis discussed in the paper can also be extended to other centralised sensor architectures (not necessarily cluster-based) that employ UWB signals, where a central node controls the assignment of TH codes to the other nodes. On the other hand, the GA framework can be applied to any asynchronous network. The authors believe that the proposed adaptation scheme can be used for cognitive communications for extending the capabilities of future wireless networks.

\section{References}

1 Win, M.Z., and Scholtz, R.A.: 'Impulse radio: how it works', IEEE Commun. Lett., 1998, 2, (2), pp. 36-38

2 Guvenc, I., and Arslan, H.: 'Design and performance analysis of time hopping sequences for UWB-IR systems'. Proc. IEEE Wireless 
Commun. Networking Conf. (WCNC), Atlanta, GA, March 2004, vol. 2, pp. 914-919

3 Guvenc, I., Arslan, H., Gezici, S., and Kobayashi, H.: 'Adaptation of multiple access parameters in time hopping UWB cluster based wireless sensor networks'. Proc. IEEE Mobile $\mathrm{Ad}$-Hoc and Sensor Systems Conf. (MASS), Ft. Lauderdale, FL, October 2004, pp. $235-244$

4 Arslan, H.: 'Adaptation techniques and the enabling parameter estimation algorithms for wireless communication systems'. Signal processing communications handbook (CRC Press, 2004)

5 Boudec, J.Y.L., Merz, R., Radunovic, B., and Widmer, J.: 'A MAC protocol for UWB very low power mobile ad-hoc networks based on dynamic channel coding with interference mitigation'. EPFL Technical Report ID: IC/2004/02, Lausanne, Switzerland, Technical Report, January 2004

6 Giancola, G., Nardis, L.D., Benedetto, M.G.D., and Dubuis, E.: 'Dynamic resource allocation in time varying ultra wideband channels'. Proc. IEEE Int. Conf. Commun. (ICC), Paris, France, June 2004, vol. 6, pp. 3581-3585

7 Zhang, H., and Kohno, R.: 'Soft-spectrum adaptation in UWB impulse radio'. Proc. IEEE Personal Indoor Mobile Radio Commun. (PIMRC), Beijing, China, September 2003, vol. 1, pp. 289-293

8 Kolenchery, S.S., Townsend, J.K., Freebersyser, J.A., and Bilbro, G.: 'Performance of local power control in peer-to-peer impulse radio networks with bursty traffic'. Proc. IEEE Global Telecommun. Conf, Phoenix, AR, November 1997, vol. 2, pp. 910-916

9 Oh, S.J., and Wasserman, K.M.: 'Adaptive resource allocation in power constrained CDMA mobile networks'. Proc. IEEE Wireless Commun. Networking Conf. (WCNC), New Orleans, LA, September 1999, vol. 1, pp. 510-514

10 Kim, D.: 'Rate-regulated power control for supporting flexible transmission in future CDMA mobile networks', IEEE J. Sel. Areas Commun., 1999, 17, (5), pp. 968-977

11 Yun, L.C., and Messerschmitt, D.G.: 'Variable quality of service in CDMA systems by statistical power control'. Proc. IEEE Int. Conf. Commun, Seattle, WA, June 1995, vol. 2, pp. 713-719

12 Berggren, F., and Kim, S.L.: 'Energy-efficient control of rate and power in DS-CDMA systems', IEEE Trans. Wirel. Commun., 2004, 3, (3), pp. $725-733$

13 Diaz, J., and Bar-ness, Y.: 'Adaptive transmission for UWB impulse radio communications'. Proc. Conf. Information Sciences Syst. (CISS), Baltimore, MD, March 2003

14 Giancola, G., Nardis, L.D., and Benedetto, M.G.D.: 'Multi user interference in power-unbalanced ultra wide band systems: analysis and verification'. Proc. IEEE Ultrawideband Syst. Technol. Conf. UWBST, Reston, VA, November 2003, pp. 325-329

15 Gezici, S., Kobayashi, H., Poor, H.V., and Molisch, A.F.: 'Performance evaluation of impulse radio UWB systems with pulse-based polarity randomization in asynchronous multiuser environments'. Proc. IEEE Wireless Commun. Networking Conf. (WCNC), Atlanta, GA, March 2004

16 Yomo, H., Popovski, P., Wijting, C., Kovacs, I.Z., Deblauwe, N., Baena, A.F., and Prasad, R.: 'Medium access techniques in ultra-wideband ad hoc networks'. Proc. 6th National Conf. Society for Electronic, Telecommun., Automatics, and Informatics (ETAI), Ohrid, Macedonia, September 2003

17 Cuomo, F., Martello, C., Baiocchi, A., and Capriotti, F.: 'Radio resource sharing for ad hoc networking with UWB', IEEE J. Sel. Areas Commun., 2002, 20, (9), pp. 1722-1732

$18 \mathrm{Zu}, \mathrm{H}$., and Ganz, A.: 'A radio resource control method in UWB MAC protocol design'. Proc. IEEE Military Commun. Conf. (MILCOM), Boston, MA, October 2003, vol. 2, pp. 886-891

19 Fishler, E., and Poor, H.V.: 'On the tradeoff between two types of processing gain'. Proc. 40th Annual Allerton Conf. Commun. Control Computing, Monticello, IL, October 2002

20 Heinzelman, W.R., Chandrakasan, A., and Balakrishnan, H.: 'Energy efficient communication protocol for wireless microsensor networks'. Proc. Annual Hawaii Int. Conf. System Sciences, Hawaii, January 2000, pp. 3005-3013

21 'Federal Communications Commision: revision of part 15 of the commision's rules regarding ultra-wideband transmission system', First Report and Order, ET Docket 98-153, FCC 02-48, April 2002

22 Gezici, S., Kobayashi, H., Poor, H.V., and Molisch, A.F.: 'Performance evaluation of impulse radio UWB systems with pulse-based polarity randomization', IEEE Trans. Signal Process., 2005, 53, (7), pp. $1-13$

$23 \mathrm{He}, \mathrm{N}$. , and Tepedelenlioglu, C.: 'Adaptive synchronization for non-coherent UWB receivers'. Proc. IEEE Int. Conf. Acoustics, Speech, and Signal Processing (ICASSP'04), Montreal, Quebec, Canada, May 2004, vol. 4, pp. 517-520

24 Kullback, S.: 'Information theory and statistics' (Wiley, New York, 1959)

25 Billingsley, P.: 'Probability and measure' (Wiley, New York, 1986, 2nd edn.)

\section{$9 \quad$ Appendix}

\subsection{Proof of Lemma 1}

From (2) and (3), the MAI from user $k, M_{k}$ in (5), can be expressed as follows

$$
M_{k}=\sqrt{\frac{E_{\mathrm{rp}}^{(k)}}{N_{\mathrm{s}}^{(\xi)}}} \sum_{l=0}^{N_{\mathrm{s}}^{(\xi)}-1} M_{k, l}
$$

where

$$
\begin{aligned}
M_{k, l}= & a_{l}^{(\xi)} \sum_{j=-\infty}^{\infty} a_{j}^{(k)} b_{\left\lfloor j / N_{\mathrm{s}}^{(k)}\right.}^{(k)} R\left(j T_{\mathrm{f}}^{(k)}-l T_{\mathrm{f}}^{(\xi)}\right. \\
& \left.+c_{j}^{(k)} T_{\mathrm{c}}-c_{l}^{(\xi)} T_{\mathrm{c}}-\Delta_{k} T_{\mathrm{c}}\right)
\end{aligned}
$$

with $\Delta_{k}=\left(\tau_{k}-\tau_{\xi}\right) / T_{\mathrm{c}}$ being the amount of asynchronism between the desired user and user $k$ in terms of the chip interval, and $R(x)=\int_{-\infty}^{\infty} w_{r x}(t+x) w_{r x}(t) \mathrm{d} t$.

First, consider the case in which $N_{\mathrm{s}}^{(\xi)} \leq N_{\mathrm{s}}^{(k)}$. It can be shown that $\left\{M_{k, l}\right\}_{l=0}^{N_{\mathrm{s}}^{(1)}-1}$ form a 1-dependent sequence [25], which means that $M_{k, l_{1}}$ and $M_{k, l_{2}}$ are independent for $\left|l_{1}-l_{2}\right|>1$. This is due to the facts that the interference to frames $l_{1}$ and $l_{2}$ of the desired user always comes from different frames of user $k$ for $\left|l_{1}-l_{2}\right|>1$ and that the random polarity codes are independent and identically distributed as binary random variables $(-1,+1)$. The random polarity codes also result in a zero mean distribution for each term of the sequence $\left\{M_{k, l}\right\}_{l=0}^{N_{\mathrm{s}}^{(7)}-1}$; that is, $\mathrm{E}\left\{M_{k, l}\right\}=0$ for $l=0,1, \ldots, N_{\mathrm{s}}^{(1)}-1$.

For a 1-dependent zero-mean sequence, the central limit theorem for dependent sequences can be applied to obtain the asymptotic distribution of $\left(1 / \sqrt{ }\left(N_{\mathrm{s}}^{(\xi)}\right)\right) \sum_{l=0}^{N_{\mathrm{s}}^{(s)}-1} M_{k, l}$, as $N_{\mathrm{s}}^{(\xi)} \rightarrow \infty$, as $\mathcal{N}\left(0, E\left\{M_{k, l}^{2}\right\}+2 E\left\{M_{k, l} M_{k, l+1}\right\}\right)[25]$.

It can be shown that the correlation terms are zero due to the fact that random polarity codes are zero mean and independent for different indices. Also after some manipulation, it can be shown that $E\left\{M_{k, l}^{2}\right\}=1 / N_{\mathrm{h}}^{(k)}$. Hence, it is obtained that

$$
\sqrt{\frac{E_{\mathrm{rp}}^{(k)}}{N_{\mathrm{s}}^{(\xi)}}} \sum_{l=0}^{N_{\mathrm{s}}^{(\mathrm{s})}-1} M_{k, l} \sim \mathcal{N}\left(0, \frac{E_{\mathrm{rp}}^{(k)}}{N_{\mathrm{h}}^{(k)}}\right)
$$

as $N_{\mathrm{s}}^{(\xi)} \rightarrow \infty$. Therefore for large $N_{\mathrm{s}}^{(\xi)}, M_{k}$ in (27) can be approximated as in (9).

For $N_{\mathrm{s}}^{(\xi)}>N_{\mathrm{s}}^{(k)}$, the MAI from user $k$ can be expressed as the summation of frame interference terms as follows

$$
M_{k}=\sqrt{\frac{E_{\mathrm{rp}}^{(k)}}{N_{\mathrm{s}}^{(\xi)}}} \sum_{l=0}^{N_{\mathrm{s}}^{(k)}} \hat{M}_{k, l}
$$

where $\hat{M}_{k, l}$ is the interference related to the $l$ th frame of user $k$

$$
\begin{aligned}
\hat{M}_{k, l}= & a_{l}^{(k)} b_{\left\lfloor l / N_{\mathrm{s}}^{(k)}\right\rfloor}^{(k)} \sum_{j=0}^{N_{\mathrm{s}}^{(\xi)}-1} a_{j}^{(\xi)} R\left(j T_{\mathrm{f}}^{(k)}-l T_{\mathrm{f}}^{(\xi)}\right. \\
& \left.+c_{j}^{(k)} T_{\mathrm{c}}-c_{l}^{(\xi)} T_{\mathrm{c}}-\Delta_{k} T_{\mathrm{c}}\right)
\end{aligned}
$$

It can be shown that $\left\{\hat{M}_{k, l}\right\}_{l=1}^{N_{\mathrm{s}}^{(k)}-1}$ form a 1-dependent sequence (As $N_{\mathrm{s}}^{(k)} \rightarrow \infty$, the edge values, $\hat{M}_{k, 0}$ and $\hat{M}_{k, N_{\mathrm{s}}^{(k)}}$ can be omitted for simplicity.). Then, as $N_{\mathrm{s}}^{(k)} \rightarrow \infty$

$$
\frac{1}{\sqrt{N_{\mathrm{s}}^{(k)}}} \sum_{l=1}^{N_{\mathrm{s}}^{(k)}-1} \hat{M}_{k, l} \sim \mathcal{N}\left(0, \frac{1}{N_{\mathrm{h}}^{(\xi)}}\right)
$$


For large $N_{\mathrm{s}}^{(k)}, M_{k}$ is approximately distributed as $\mathcal{N}\left(0, E_{\mathrm{rp}}^{(k)}\left(N_{\mathrm{s}}^{(k)} / N_{\mathrm{s}}^{(\xi)} N_{\mathrm{h}}^{(\xi)}\right)\right)$. However, since the total gain $N_{\mathrm{c}}$ is constant for all users; that is, $N_{\mathrm{s}}^{(k)} N_{\mathrm{h}}^{(k)}=N_{\mathrm{s}}^{(\xi)} N_{\mathrm{h}}^{(\xi)}$, the variance is the same as that in (9).

All in all, for large values of $\min \left\{N_{\mathrm{s}}^{(k)}, N_{\mathrm{s}}^{(\xi)}\right\}$, the distribution of the MAI from user $k$ is approximately given by (9).

\subsection{Proof of Lemma 2}

The proof is similar to the proof in 9.1. First, consider the case in which $N_{\mathrm{s}}^{(\xi)}<N_{\mathrm{s}}^{(k)}$. The MAI from user $k$ can be expressed, from (19) to (23), as

$$
M_{k}=\sqrt{\frac{E_{\mathrm{rp}}^{(k)}}{N_{\mathrm{s}}^{\xi}}} \sum_{j=0}^{N_{\mathrm{s}}^{(\mathrm{s})}-1} M_{k, j}
$$

where

$$
\begin{aligned}
M_{k, j}= & a_{j}^{(1)} \sum_{m=-\infty}^{\infty} a_{m}^{(k)} b_{\left\lfloor m / N_{\mathrm{s}}^{(k)}\right.}^{(k)} \phi_{u v}^{(k)}\left(m T_{\mathrm{f}}^{(k)}-j T_{\mathrm{f}}^{(1)}\right. \\
& \left.+\left(c_{m}^{(k)}-c_{j}^{(1)}\right) T_{\mathrm{c}}+\Delta_{k} T_{\mathrm{c}}\right)
\end{aligned}
$$

with $\phi_{u v}^{(k)}(x)$ being defined as

$$
\phi_{u v}^{(k)}(x)=\int_{-\infty}^{\infty} u^{(k)}(t-x) v(t) \mathrm{d} t
$$

Note that $\left\{M_{k, j}\right\}_{j=0}^{N_{\mathrm{s}}^{(\mathrm{s})}}$ forms a 1-dependent sequence [25] since it is assumed that the delay spreads of the channels are smaller than the frame intervals, which results in different interference terms for each pair of interference terms $M_{k, j_{1}}$ and $M_{k, j_{2}}$ for $\left|j_{1}-j_{2}\right|>1$.

Owing to the distribution of the polarity codes, the mean and correlation terms can be shown to be zero; that is, $E\left\{M_{k, j} \mid \Delta_{k}\right\}=0$, and $E\left\{M_{k, j} M_{k, l} \mid \Delta_{k}\right\}=0$ for $l \neq j$. In order to calculate the variance, the facts that the polarity codes are independent for different user and frame indices and that the $\mathrm{TH}$ sequences are uniformly distributed are employed. Then, it can be shown that

$$
\begin{aligned}
E\left\{M_{k, j}^{2} \mid \Delta_{k}\right\}= & \frac{1}{N_{\mathrm{h}}^{(\xi)} N_{\mathrm{h}}^{(k)}} \sum_{i=0}^{N_{\mathrm{h}}^{(k)}-1} \sum_{l=0}^{N_{\mathrm{h}}^{(\xi)}-1} \sum_{m=-\infty}^{\infty} \times\left\{\phi _ { u v } ^ { ( k ) } \left(m T_{\mathrm{f}}^{(k)}\right.\right. \\
& \left.\left.-j T_{\mathrm{f}}^{(\xi)}+(i-l) T_{\mathrm{c}}+\Delta_{k} T_{\mathrm{c}}\right)\right\}^{2}
\end{aligned}
$$

which reduces, after some manipulation, to

$$
E\left\{M_{k, j}^{2} \mid \Delta_{k}\right\}=\frac{1}{N_{\mathrm{h}}^{(k)}} \sum_{j=-(L-1)}^{L-1}\left[\phi_{u v}^{(k)}\left(j T_{\mathrm{c}}\right)\right]^{2}
$$

From (35), (20) and (22), (37) can be expressed as

$$
\begin{aligned}
E\left\{M_{k, j}^{2} \mid \Delta_{k}\right\}= & \frac{1}{N_{\mathrm{h}}^{(k)}}\left[\sum_{j=1}^{L}\left(\sum_{i=1}^{j} \beta_{i} \alpha_{i-j+L}^{(k)}\right)^{2}\right. \\
& \left.+\sum_{j=1}^{L-1}\left(\sum_{i=1}^{j} \alpha_{i}^{(k)} \beta_{i-j+L}\right)^{2}\right]
\end{aligned}
$$

Note that the result is independent of the offset $\Delta_{k}$. Therefore $E\left\{M_{k, j}^{2}\right\}$ is given by (38), as well.

Since $\left\{M_{k, j}\right\}_{j=0}^{N_{\mathrm{s}}^{(\xi)}-1}$ is a 1 -dependent sequence with zero mean and correlation terms, the distribution of $\sqrt{ }\left(\left(E_{\mathrm{rp}}^{(k)} / N_{\mathrm{s}}^{(\xi)}\right)\right) \sum_{j=0}^{N_{\mathrm{s}}^{(\xi)}-1} M_{k, j}$ converges to the Gaussian distribution given by $\mathcal{N}\left(0, E_{\mathrm{rp}}^{(k)} E\left\{M_{k, j}^{2}\right\}\right)$, as $N_{\mathrm{s}}^{(\xi)} \rightarrow \infty$ [25]. Then, (24) follows from (38) for the $N_{\mathrm{s}}^{(\xi)}<N_{\mathrm{s}}^{(k)}$ case.

For $N_{\mathrm{s}}^{(\xi)} \geq N_{\mathrm{s}}^{(k)}$, the MAI from user $k$ can be expressed as $M_{k}=\sqrt{ }\left(E_{\mathrm{rp}}^{(k)} / N_{\mathrm{s}}^{(\xi)}\right) \sum_{j=0}^{N_{\mathrm{s}}^{(k)}} \hat{M}_{k, j}$, where $\hat{M}_{k, j}$ denotes the interference related to the $j$ th frame of user $k$. Then, by similar arguments, (24) can be derived.

Therefore the result in Lemma 2 is obtained as $\min \left\{N_{\mathrm{s}}^{(\xi)}, N_{\mathrm{s}}^{(k)}\right\} \rightarrow \infty$. 